\title{
EFFECT OF SEN IMMERSION DEPTH ON MOLD FLOW PROFILE AND SLAG ENTRAPMENT DURING CONTINUOUS CASTING OF STEEL
}

\author{
Mallikarjuna Rao $P^{l *}$, Vaibhav Vasantrao Chougale ${ }^{2}$, Satish Kumar $D^{l}$, \\ Rajendra $T^{l}$, Balachandran $G^{l}$ \\ ${ }^{1}$ JSW Steel Limited, Vijayanagar Works, Toranagallu, Bellary, Karnataka, India. \\ ${ }^{2}$ College of Engineering, Shivajinagar, Pune, Maharashtra, India.
}

Received 31.05.2021

Accepted 13.07.2021

\begin{abstract}
Mold flux entrapment during continuous casting of steel contributes to both surface and sub-surface defects in the final product. Continuous casting operating parameters such as casting speed, SEN immersion depth, SEN port geometry, argon flow, and mold EMS significantly affect the mold flow conditions and flow profile. During continuous casting operation, SEN immersion depth is continuously varied to avoid localized erosion of SEN, and it impacts the flow dynamics in the mold. In the present work, water modeling studies were carried out for a wide range of mold widths (1200-1800 mm) and casting speeds $(0.8-1.4 \mathrm{~m} / \mathrm{min})$ on a 0.5 scaled down water model to optimize casting speed for different combinations of SEN immersion depth and mold width. Results from water modeling were further validated using nail board studies in the actual plant. A safe operating matrix was identified from these experiments to avoid mold slag entrapment during continuous casting.
\end{abstract}

Keywords: Mold slag entrapment; SEN immersion depth; Casting speed; Mold flow profile; Water modeling; Nail board.

\section{Introduction}

Ultra-low carbon and interstitial free (IF) steels are widely used in automobiles and appliances due to their excellent deep drawability. Non-metallic inclusions and entrapped mold fluxes during continuous casting adversely affect the quality of these steels and contribute to diversions. Mold flux entrapment during continuous casting of slabs contributes to both surface and sub-surface defects in the final product. Mold flux and argon bubbles can be entrained into solidifying steel shell during excessive mold level

${ }^{*}$ Corresponding author: Mallikarjuna Rao P, mallikarjunrao.panabaka@jsw.in 
fluctuations induced by abnormal casting conditions. Mold powder and inclusions present in the slab surface layer are closely related to the initial solidification and the flow of liquid steel in the meniscus region of the mold [1]. Therefore, the flow profile of liquid steel in the continuous casting mold plays a vital role in controlling the mold slag entrainment.

Mold slag gets entrained in continuous cast slabs due to high mold level fluctuations, shear layer instability, meniscus freezing, vortexing, argon bubble interactions, narrow face spout impingement upon the top surface, and slag crawling down the submerged entry nozzle [2]. However, for each mechanism, there exists a critical condition that can be evaluated by various methods, and a safe operating window can be identified from these methods to avoid mold slag entrapment. Mold flow profile also affects the initial solidification and mold powder lubrication behavior between mold wall and solidifying shell. High mold level fluctuations and large meniscus velocities caused by unfavorable flow profiles lead to mold powder entrapment. Mold flow profile in continuous casting is affected by submerged entry nozzle (SEN) size, SEN port geometry, casting speed, mold width, mold thickness, SEN submergence depth, argon flow rate, and electromagnetic stirring (EMS). However, in actual plant continuous casting operation, SEN submergence depth is continuously varied between 200 to 100 $\mathrm{mm}$ in steps to avoid the localized erosion of SEN. Under this condition, at any mold width, certain SEN immersion depth and casting speed combinations may create unfavorable flow conditions, which will contribute to a change in mold flow from double roll flow to single roll (or) mixed flow. Single roll (or) mixed flow increases the chances of mold slag entrapment in slabs that finally contribute to sliver defects in HR/CR coils. The surface (meniscus) velocity of the continuous casting mold is considered as an indirect indication of slag entrapment, and velocities greater than a critical value promote the slag entrapment [2]. The effect of casting speed and mold width on mold flow pattern and slag entrapment has been studied extensively by water modeling, nail boards, refractory paddles, velocity sensors, particle image velocimetry (PIV), mathematical simulation etc. However, the influence of SEN immersion depth along with casting speed and mold width on mold flow profile and slag entrapment is less investigated.

In the present study, the effect of SEN immersion depth at different casting speeds and mold widths on mold flow profile and mold slag entrapment was investigated using laboratory water modelling studies and validated with nail board experiments at the plant continuous caster. An optimum operating window with respect to SEN immersion depth was identified in this study to avoid mold slag entrapment and reduce mold related slab defects.

\section{Experimental}

Water modeling is one of the most versatile techniques for simulating molten steel flow under laboratory conditions. This technique is also useful to visualize the flow profile inside the steel making vessels, which is impossible under the actual plant operating conditions. Water modeling studies can be performed under full scale or reduced scale, satisfying the geometrical, dynamic, and kinematic similarities between the actual plant conditions and the model. Water can be used to simulate molten steel flow in water modeling owing to the fact that the kinematic viscosity of water at room temperature is very similar to that of molten steel at steelmaking temperatures and hence exhibits a similar flow behavior. 
The water modelling experimental setup used in the present study is shown in Fig. 1. The setup is an acrylic glass scaled down model with a 0.5 scale factor and consists of the continuous casting mold, submerged entry nozzle (SEN) with $25^{\circ}$ downward port angle, flow control valve, rotameter to measure the flow of water, water circulation pump, and a water storage tank. The setup has a tracer injection point as indicated in Fig.1 to inject tracer (dye) for the visualization of mold flow under different experimental conditions.

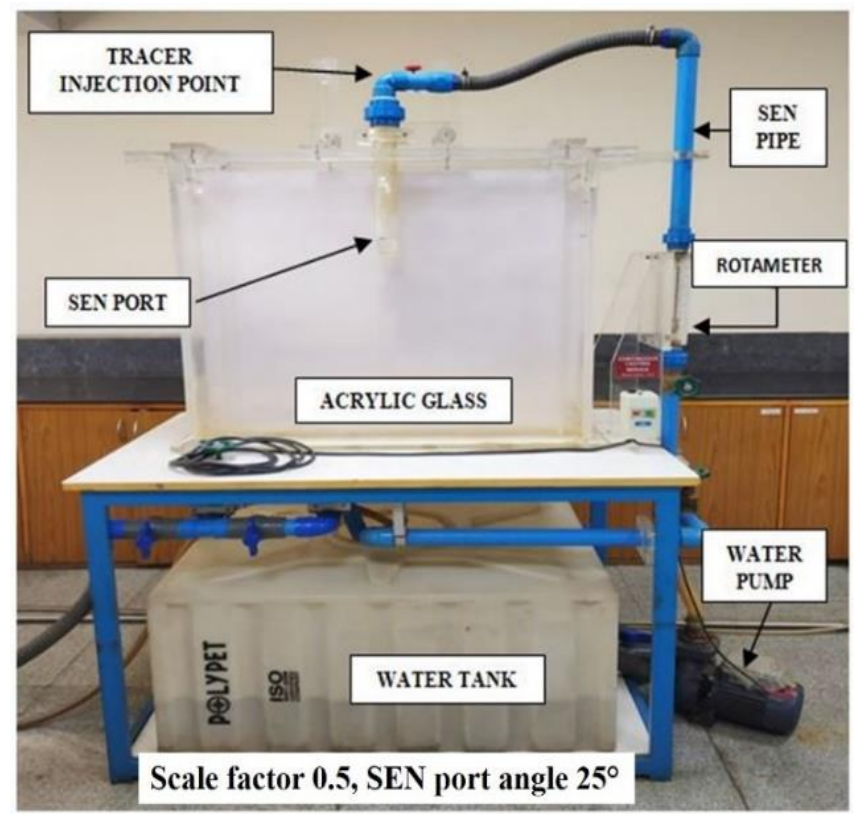

Fig. 1. Water modeling setup.

Experimental flow rates for water modeling were calculated from the throughput of caster corresponding to casting speeds $(0.8,1.0,1.2$, and $1.4 \mathrm{~m} / \mathrm{min})$ and mold widths $(1200,1400,1600$, and $1800 \mathrm{~mm})$. These experimental flow rates were calculated according to equation (1), considering the geometrical and dynamic similarity between the water model and the actual casting flow rates [3]

$$
\frac{Q_{m}}{Q_{p}}=\lambda^{2.5}
$$

In equation (1), $\mathrm{Q}_{\mathrm{m}}$ is the flow rate of water in the water model, $\mathrm{Q}_{\mathrm{p}}$ is the flow rate of steel in mold (throughput), and $\lambda$ is the scale factor of the model.

During the continuous casting of steel, SEN is gradually eroded at mold slag-SEN interfacial area due to the reaction of liquid slag with SEN material, as shown in Fig. 2. Therefore, in actual operation, SEN immersion depth is varied between 200 to $100 \mathrm{~mm}$, as shown in Fig. 3, to avoid localized erosion of zirconia coating on SEN. This variation in SEN immersion depth significantly affects the mold flow profile and promotes mold slag entrapment under a certain combination of casting speed and mold width. Therefore, water model experimental parameters are selected to evaluate the effect of SEN 
immersion depth, casting speed, and mold width on the mold flow profile and slag entrapment and presented in Table 1. All water model experiments were conducted under steady state conditions. Mustard oil (density $910 \mathrm{~kg} / \mathrm{m}^{3}$ ) with 7-8 mm thickness was used in water model experiments to replicate mold slag. A small quantity of aqueous solution of potassium permanganate $\left(\mathrm{KMnO}_{4}\right)$ was injected into the flowing stream of water as a dye in each experiment, and the trajectory of the dye was recorded using a high speed digital camera.

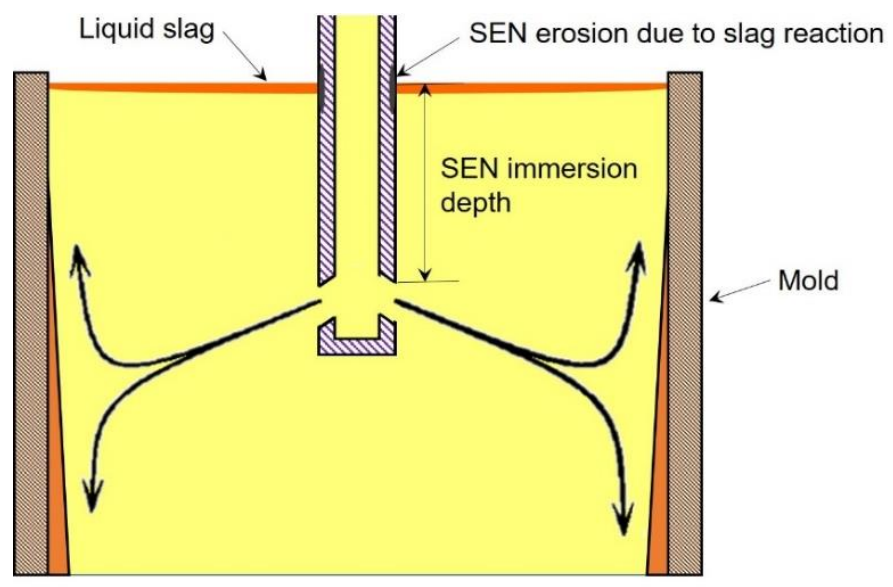

Fig. 2. Schematic diagram of continuous casting mold showing SEN erosion.

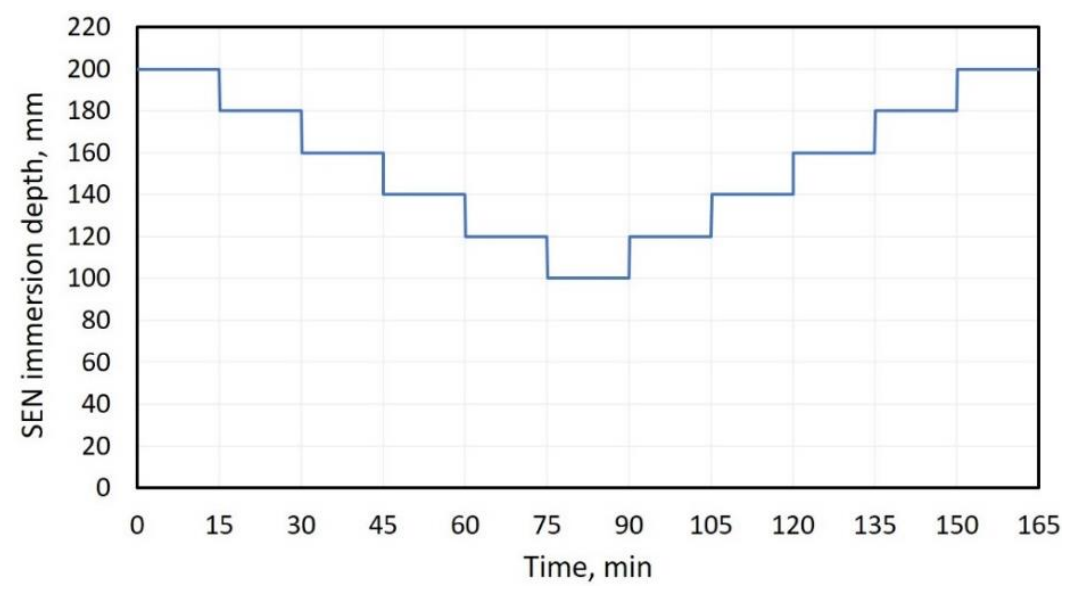

Fig. 3. The variation of SEN immersion depth during continuous casting of steel to avoid SEN localized erosion. 
Table 1. Water modeling experimental parameters.

\begin{tabular}{lll}
\hline & Plant & Water model \\
\hline Scale factor $(\lambda)$ & 1 & 0.5 \\
Mold thickness, mm & 220 & 110 \\
Mold height, mm & 1000 & 500 \\
Mold width, mm & $1200,1400,1600 \& 1800$ & $600,700,800 \& 900$ \\
Steel/water flow rate & $1.658-4.352$ ton/min & $37-98 \mathrm{lpm}$ \\
SEN submergence depth, & $100,120,160 \& 200$ & $50,60,80 \& 100$ \\
mm & & \\
SEN port dimensions, mm & $75 \times 95$ & $37.5 \times 47.5$ \\
SEN port downward angle & $25^{\circ}$ & $25^{\circ}$ \\
\hline
\end{tabular}

\section{Results and discussions}

Mold flow profile from water modelling experiments for mold width $1200 \mathrm{~mm}$, casting speed $1.4 \mathrm{~m} / \mathrm{min}$, and SEN immersion depths $100 \mathrm{~mm}$ is shown in Fig. 4. Flow patterns obtained under all experimental conditions of water modeling exhibited typical double roll flow where liquid jet exiting submerged entry nozzle (SEN) strikes the narrow face of the mold and splits into two loops. One loop travels up to the meniscus along the narrow wall and then flows towards SEN, forming an upper roll flow. Meanwhile, the other loop forms a lower roll flow that travels downwards. Medium strong double roll flow is desirable to avoid mold slag entrapment, large mold level fluctuations, thinning of top molten slag layer, and loss of lubrication between copper mold wall and solidifying steel shell.

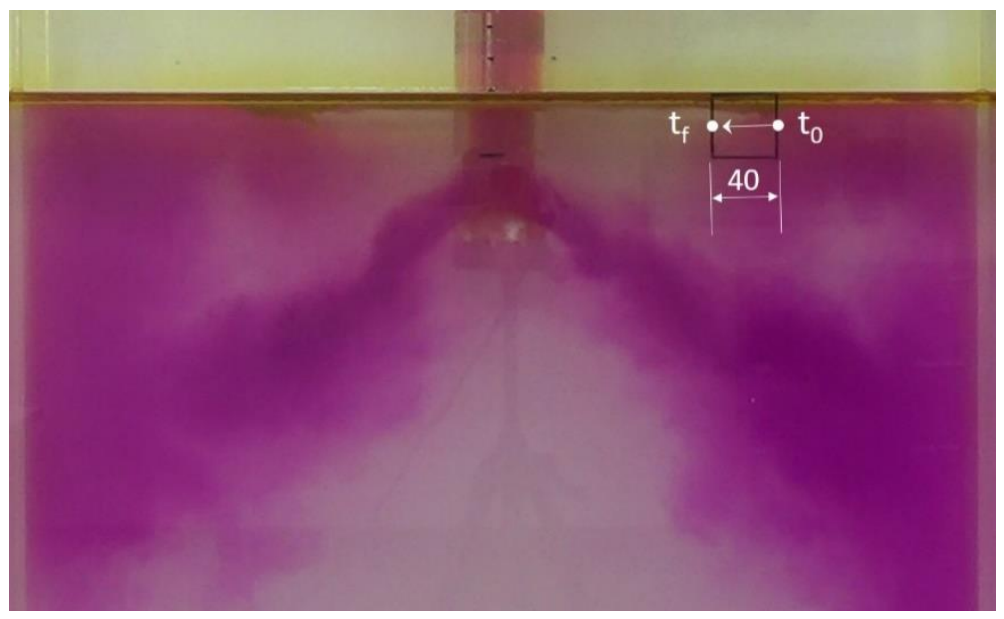

Fig. 4. Flow profile from water modeling experiments for mold width $1200 \mathrm{~mm}$, SEN immersion depth $100 \mathrm{~mm}$ and casting speed $1.4 \mathrm{~m} / \mathrm{min}$. The location for the measurement of surface velocity at mold quarter width is also indicated. 
A square box of $40 \times 40 \mathrm{~mm}$ size was marked on the model at quarter width, as shown in Fig. 4, to measure meniscus velocity. Meniscus velocity at each experimental condition was calculated from equation (2) as the time taken to travel the injected dye at a distance of $40 \mathrm{~mm}$, as indicated in Fig. 4.

$$
\text { Meniscus velocity }=\frac{40}{1000 \times\left(t_{f}-t_{0}\right)} \mathrm{m} / \mathrm{sec}
$$

In equation (2), $t_{0}$ and $t_{f}$ are dye entry and exit times (milliseconds) respectively across the marked square box.

In double roll mold flow, mold level fluctuations, shear layer instability, and vertex formation are strongly correlated with the strength of the upward flow at the narrow face. Exiting jet collision velocity at the narrow face increases with increasing the casting speed and decreasing slab width. The depth of impingement point on the narrow face increases with an increase in slab width, increasing SEN immersion depth and decreasing the argon flow rate [2]. The effect of casting speed and SEN depth on meniscus (surface) velocity is shown in Fig. 5 for mold widths 1200, $1400 \mathrm{~mm}$, and Fig. 6 for 1600, $1800 \mathrm{~mm}$. Surface (meniscus) velocity for lower mold widths (1200 and $1400 \mathrm{~mm})$ is nearly unchanged up to casting speed $1 \mathrm{~m} / \mathrm{min}$ and then increased for all SEN immersion depths, as shown in Fig. 5. However, for higher mold widths, surface velocity increased continuously with an increase in casting speed for all SEN immersion depths (Fig 6). This is because the larger mold widths lead to higher throughputs which increase SEN exit velocities. An increase in SEN exit velocity with the increase in casting speed does not necessarily cause higher surface velocities at deeper SEN immersion depths. This is due to the fact that the distance of jet to travel from SEN port exit to meniscus increases with an increase in SEN immersion depth, and hence, the momentum of jet diffuses more at meniscus resulting in lower meniscus velocities [4]. This is evident from Figs 5 and 6 as mold surface velocities at all casting speeds increased with a decrease in SEN immersion depth for all mold widths. Lower surface velocities observed at higher SEN immersion depth assists in stabilizing double roll flow (DRF) in the continuous casting mold. However, too deeper SEN immersion depths may result in thinner slag layer thickness and larger meniscus hooks which can capture large sized inclusions [2,5].

In continuous casting mold, excessive meniscus velocities contribute to surface turbulence, vortex formation, flow instability, uneven mold slag distribution, and slag entrainment [6]. Too low surface velocities result in excessive cooling near the meniscus regions, which may cause hook formation, non-uniform slag consumption, mold slag entrapment, and inclusion particles (or) bubbles leading to various surface defects [7]. The interface between mustard oil (liquid slag) and water (liquid steel) becomes unstable when the velocity difference between two fluids exceeds a critical value. This critical velocity depends on interfacial tension, density, and viscosity of mustered oil (liquid slag) and water (liquid steel). 

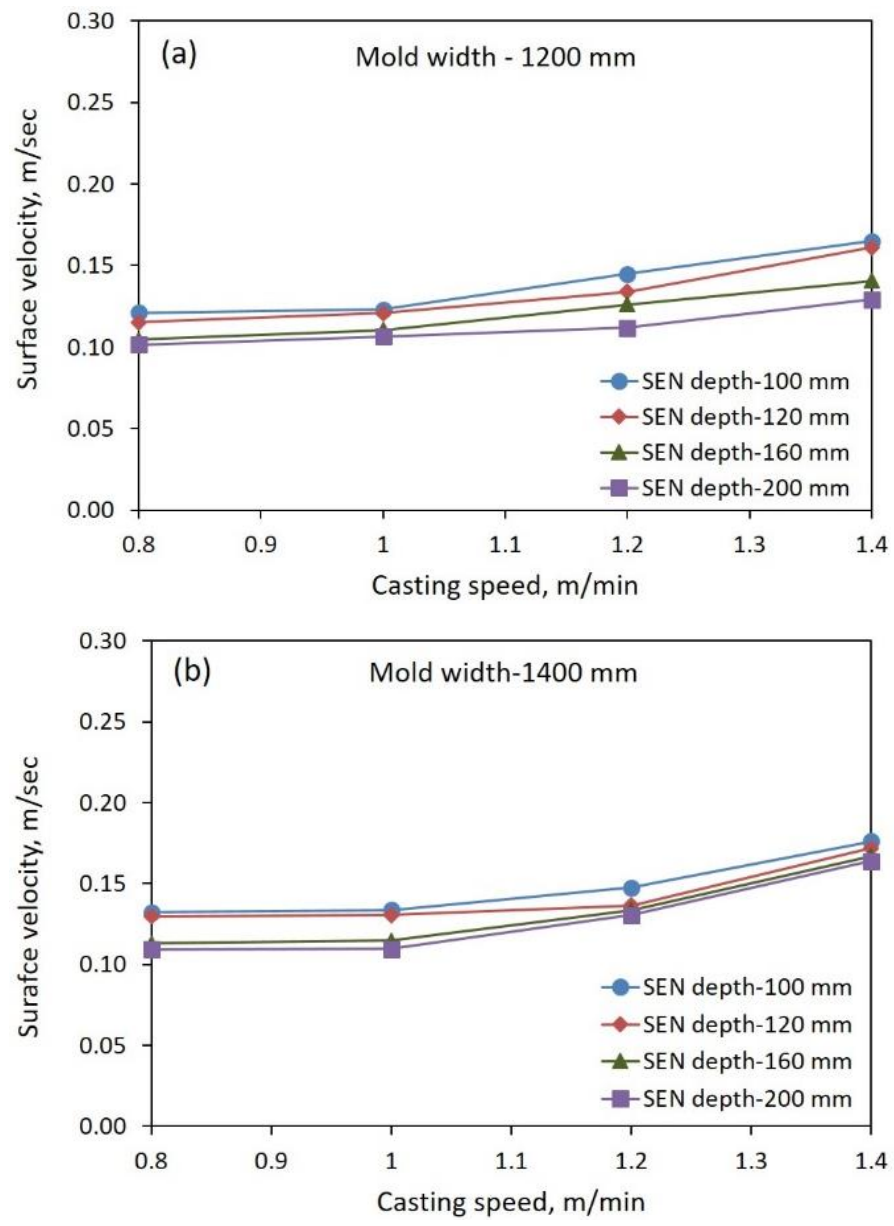

Fig. 5. Effect of casting speed and SEN immersion depth on surface velocity for mold widths (a) $1200 \mathrm{~mm}$ and (b) $1400 \mathrm{~mm}$. 

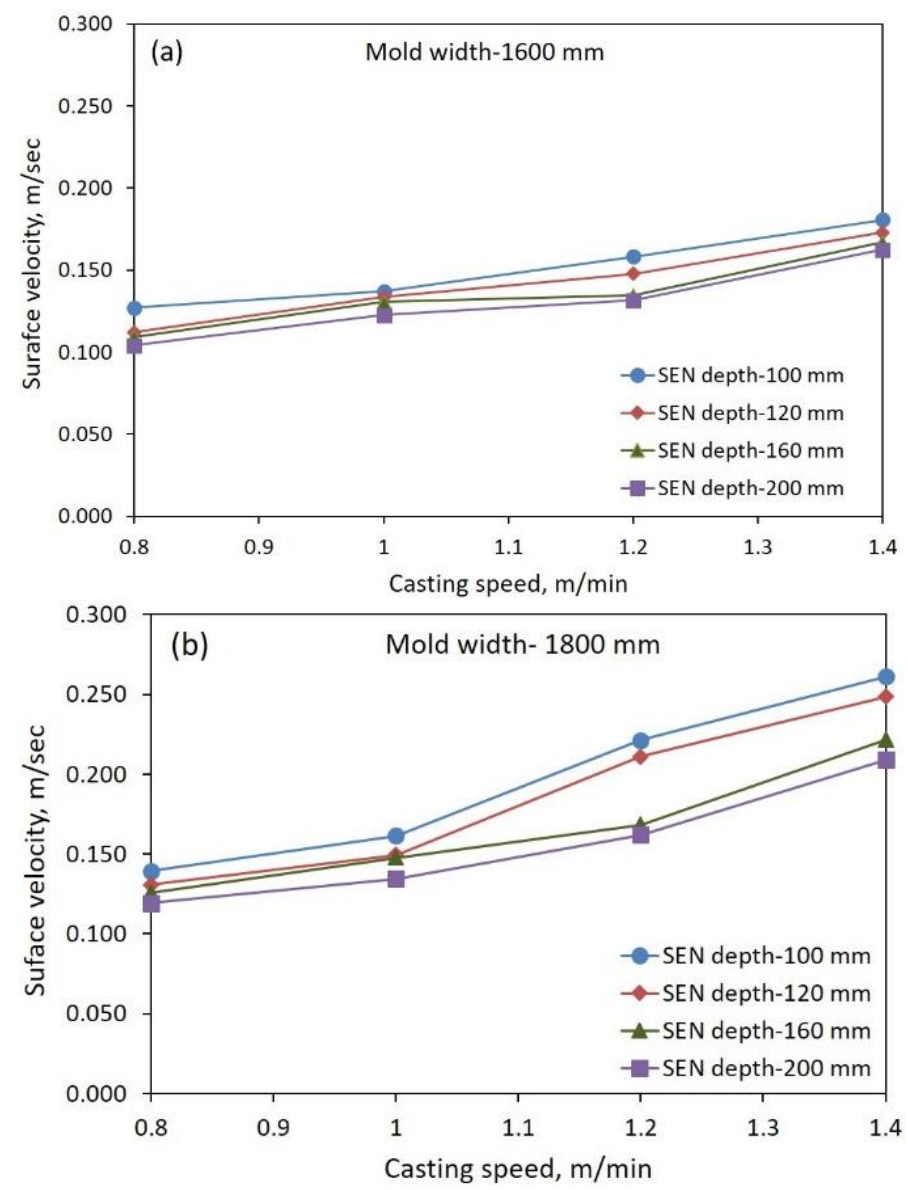

Fig. 6. Effect of casting speed and SEN immersion depth on surface velocity for mold widths (a) $1600 \mathrm{~mm}$ and (b) $1800 \mathrm{~mm}$.

Mold slag entrainment due to shear instability is most likely to occurs nearly at the mid-distance of SEN and narrow face of the mold where horizontal surface velocity is maximum. This kind of surface flow gives rise to a shearing force on the slag layer at the steel-slag interface making slag thin and drag the liquid slag downstream in the form of droplets leading to slag entrapment [2]. However, most of the slag droplets entrained into the molten steel are recaptured quickly back into the slag layer due to their buoyancy. Slag entrainment into the mold by shear layer instability is presented in Figs. 7 (a) and (b) for mold widths 1600 and $1800 \mathrm{~mm}$ respectively at casting speed $1.4 \mathrm{~m} / \mathrm{min}$ and SEN immersion depth $200 \mathrm{~mm}$. In this mechanism, an interfacial drag force is created by sufficiently high meniscus velocities that pull the top mold slag into the main stream, and the liquid slag droplets get trapped into the mold by necking and breaking of liquid slag $[8,9]$. Downward flow velocities typical of single roll flow suspend the rising slag particles in front of solidifying interface to produce slag entrainment defects. Therefore, 
single roll flow is undesirable, which can cause mold slag entrapment and adversely affect the shell lubrication.

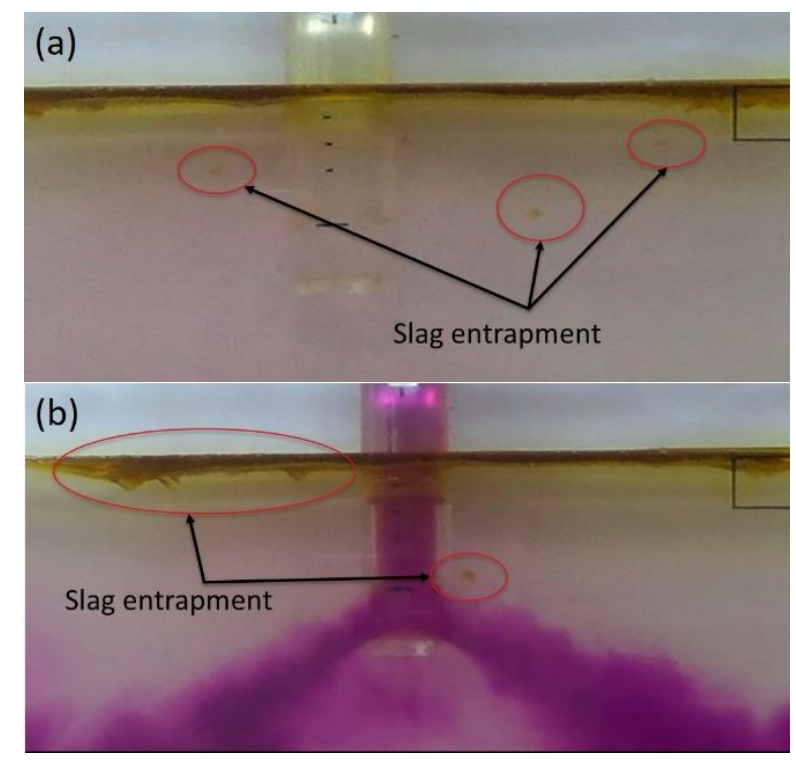

Fig. 7. Slag entrapment by shear layer instability at casting speed $1.4 \mathrm{~m} / \mathrm{min}$ and SEN immersion depth $200 \mathrm{~mm}$ for (a) mold width $1600 \mathrm{~mm}$ and (b) mold width $1800 \mathrm{~mm}$.

The tendency of mold slag entrapment from water modelling experiments for the mold widths 1200 and $1800 \mathrm{~mm}$ is shown in Figs. 8 (a) and (b) respectively. Slag entrapment was observed at casting speed $1.4 \mathrm{~m} / \mathrm{min}$ for all SEN immersion depths for mold width $1200 \mathrm{~mm}$, as shown in Fig. 8 (a). However, for $1800 \mathrm{~mm}$ mold width (Fig. 8 (b)), slag entrapment was observed at $1.4 \mathrm{~m} / \mathrm{min}$ for all SEN immersion depths and also at $1.2 \mathrm{~m} / \mathrm{min}$ for shallow SEN depths (100 and $120 \mathrm{~mm}$ ). This is due to the fact that large surface velocities are created by too strong a double roll flow (DRF) at higher casting speeds that will cause mold slag entrapment. The higher the casing speed, the easier it is to expose the meniscus near the narrow face, and slag gets entrapped by the shearing flow. In addition, with an increase of casting speed, the kinetic energy of the turbulent flow and asymmetry of the flow field in the mold is increased, and the probability of vortex formation also increases [2].

Slag on the surface layer of the mold becomes unstable with a decrease in SEN submersion depth and is prone for entrapment, as shown in Fig. 8 (b) for mold width 1800 $\mathrm{mm}$. At shallow depths $100 \mathrm{~mm}$ and $120 \mathrm{~mm}$, the slag layer near the narrow face of the mold becomes thin, and the molten steel is easy to be exposed because of a stronger impact of the liquid steel stream on the surface. By the action of the shearing force of the liquid steel stream, the molten slag is pushed from the narrow face of the mold towards SEN. This also increases the meniscus velocity and surface level fluctuation in the mold. Therefore, it could be concluded from water modelling results that higher meniscus velocities resulted from higher casting speeds and shallow SEN immersion depths 
promotes slag entrapment. These conditions are to be avoided to reduce the instances of mold slag entrapment, and subsequent hot rolled/cold rolled coil sliver instances.
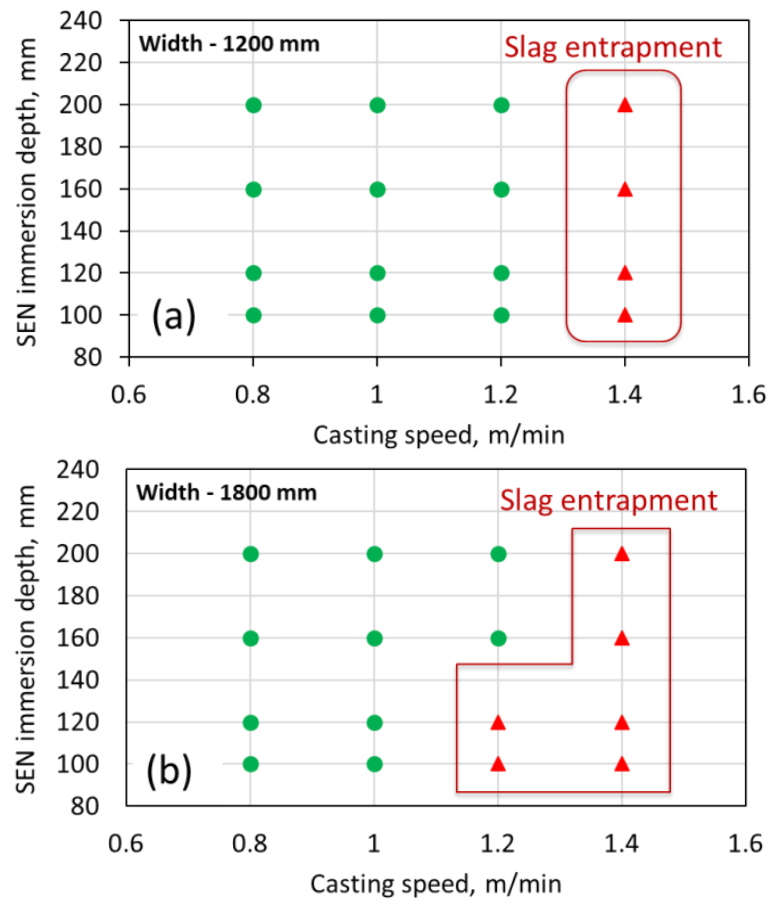

Fig. 8. Slag entrapment conditions for mold widths (a) $1200 \mathrm{~mm}$ and (b) $1800 \mathrm{~mm}$.

\section{Nail Board Experiments}

Nail board measurements are commonly used in continuous caster to measure the thickness of the molten slag layer, meniscus (surface) velocities, and mold flow profile. The results of the water model were validated by the nail board experiments in plant. A photograph of a fully assembled nail board used in the present study for mold width 1200 $\mathrm{mm}$ is shown in Fig. 9. The nail board consists of two rows of steel and aluminum nails inserted into a wooden board having the arrangement of a handle at the top. Steel and aluminum nails having each $5 \mathrm{~mm}$ diameter and $150 \mathrm{~mm}$ length are inserted into a wooden board with a spacing of $100 \mathrm{~mm}$. Nail boards are generally prepared by considering slab width, slab thickness, SEN diameter, and sensors positioned in the mold so that the board can cover the maximum surface for the experiment without disturbing the mold setup. Nail boards are designed for this study to maintain a $10-15 \mathrm{~mm}$ gap towards SEN and a 50-100 $\mathrm{mm}$ gap between the board and narrow face of the mold when inserted. 


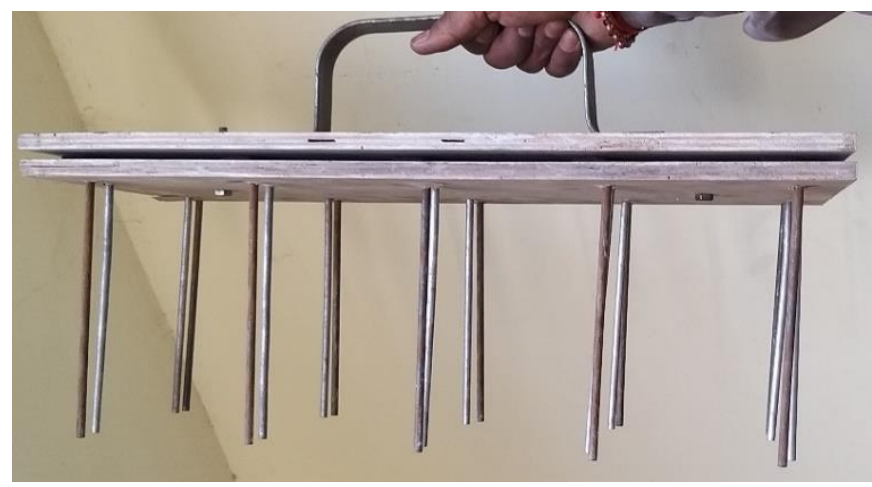

Fig. 9. Photograph of nail board for mold width of $1200 \mathrm{~mm}$.

Nail board experiments were conducted for mold widths $1200 \mathrm{~mm}$ and $1800 \mathrm{~mm}$ for casting speeds $0.8-1.4 \mathrm{~m} / \mathrm{min}$ and SEN immersion depths $100-200 \mathrm{~mm}$. These experiments were conducted by dipping nails straight down through the slag layer into the top surface of the mold between SEN and narrow face, as shown in Fig. 10. Flow velocity at the outer side of the mold (turret side) is measured from nail numbers 1-5, and nail numbers 6-10 gives flow velocity at the inner (operator) side of the mold, as indicated in Fig. 10.

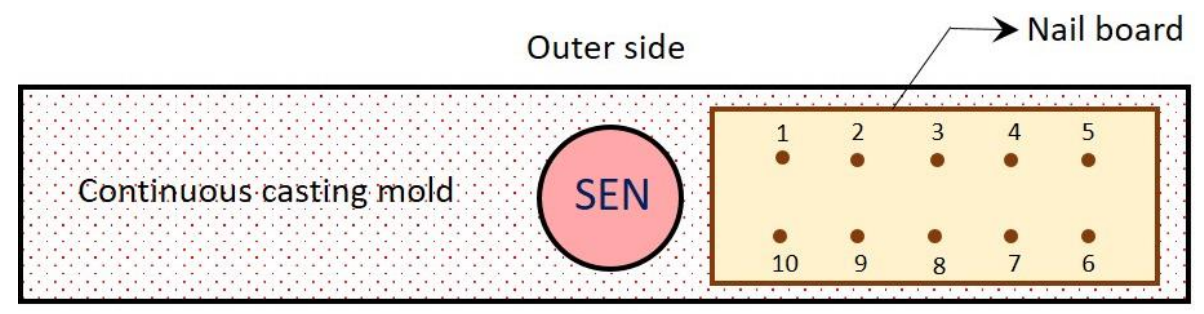

Inner side

Fig. 10. Schematic diagram (top view) showing the location of nail board insertion in mold.

Nail boards were kept immersed in the mold for 3-5 sec to form a thin skull of metal on each nail. Excessive immersion time may cause problems such as remelting of skull or melting of steel nail itself into the mold [10]. The aluminum nail melts at the top of the slag layer, and the steel skull freezes up to the steel meniscus. The difference between the two lengths gives the thickness of the molten slag layer. As illustrated in Fig. 11 , when the molten steel flows across the nail, it raises on the flowing side and drops on the other side. This is due to the fact that the kinetic energy of the impinging stream is converted into potential energy at the stagnation point (nail) and raises over the nail. The liquid steel level drops at the opposite (downstream) side of the nail due to the lower pressure. The direction and height of raise (lump height difference) $\Delta \mathrm{h}$ as marked in Fig. 11 (b) is noted for each nail of the board to calculate the direction and magnitude of stream velocity at each point. Surface velocity is then calculated by Bernoulli's equation as velocity $=\sqrt{2 g(\Delta h)} \mathrm{m} / \mathrm{sec}$, where $\mathrm{g}$ is the acceleration due to gravity $\left(\mathrm{m}^{2} / \mathrm{sec}\right)$ and $\Delta \mathrm{h}$ is lump height difference in meters. 

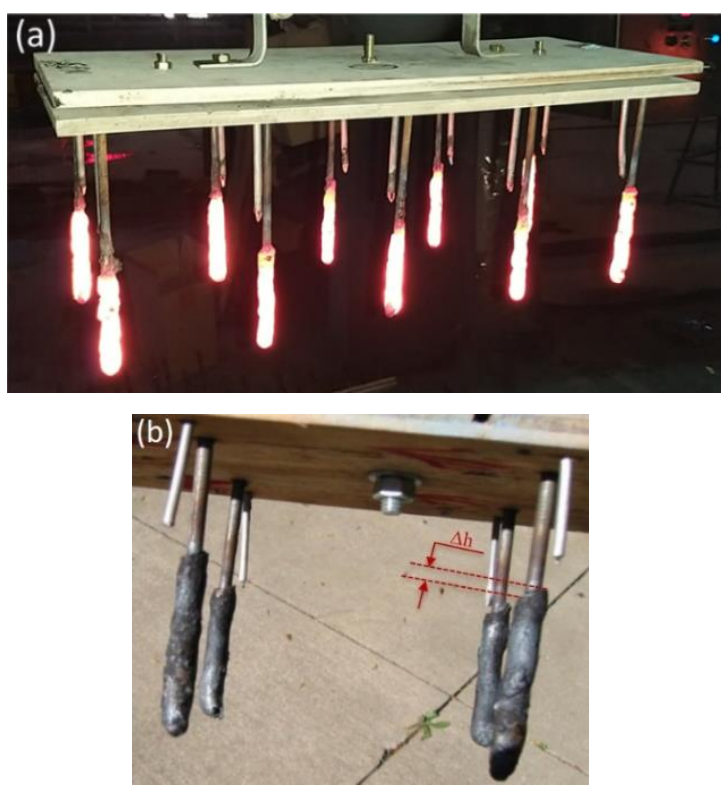

Fig. 11. (a) Nail board after dipping into the mold with solidifying skull (b) measurement of the difference in lump height $(\Delta h)$.

Nail board experiments provide valuable information about mold flow characteristics like molten slag layer thickness, meniscus flow profile, the direction of steel flow at the meniscus, and intensity of flow velocity at the meniscus. Surface (meniscus) velocity profile of mold on both inner and outer side (Fig. 10) is plotted against distance from SEN outer edge in Fig. 12 for mold width $1200 \mathrm{~mm}$, casting speed $0.8 \mathrm{~m} / \mathrm{min}$, and SEN immersion depth $200 \mathrm{~mm}$. It could be seen from Fig. 12 that surface velocities at both inner and operator side are nearly equal and reaches to a maximum value at half distance between SEN and narrow face of the mold. Mold slag entrainment is most likely to occur at this location where horizontal surface velocity is maximum, and this has already been confirmed from water modeling results presented in Fig. 7.

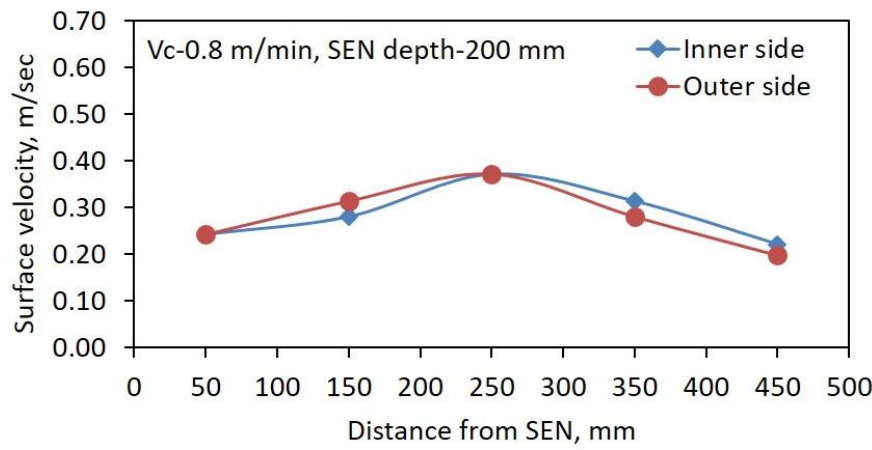

Fig. 12 Surface velocity profile along half-width of mold for casting speed $0.8 \mathrm{~m} / \mathrm{min}$, SEN immersion depth $200 \mathrm{~mm}$ for mold width $1200 \mathrm{~mm}$. 
The average velocity measured from nail board experiments is plotted against distance from SEN for casting speeds $1.4 \mathrm{~m} / \mathrm{min}$ and is presented in Fig. 13 for mold width $1200 \mathrm{~mm}$ and $1800 \mathrm{~mm}$. It could be seen from these figures that surface velocity reaches its maximum at a nearly mid location of SEN and narrow face of the mold. This maximum velocity is higher for shallow SEN immersion depths for all casting speeds and mold widths. Higher surface velocity at shallow SEN immersion depth makes the top mold slag unstable and promotes slag entrapment. However, surface velocities measured from nail board experiments are few orders of magnitude higher than those measured by the water modeling, as shown in Fig. 14. This is due to the fact that tapering of shell and subsequent reduction in fluid cross-sectional area in actual mold provides resistance for fluid flow and yields higher velocities at mold top surface. Argon purging through SEN also contributes to the increase in surface velocity of the continuous casting mold. Therefore, lower mold surface velocities in water modeling than nail board experiments are due to the absence of solidifying shell and argon purging in water modeling. However, both water modeling and nail board methods exhibited a similar trend of casting speed and SEN immersion depth on mold surface velocity.
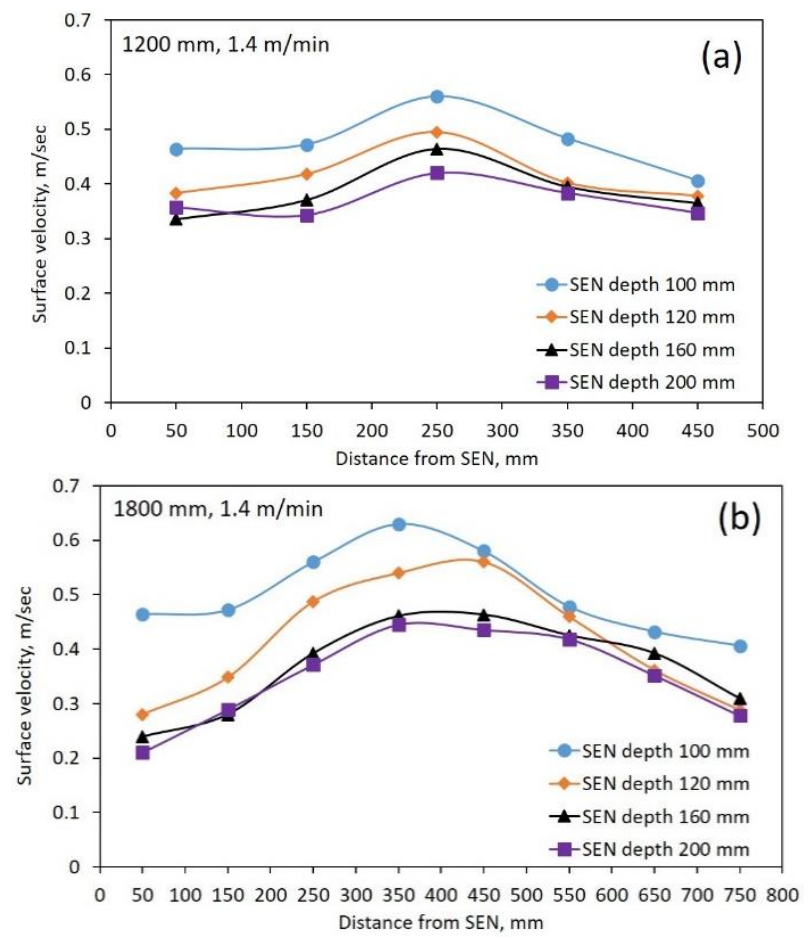

Fig. 13. The variation of meniscus velocity against the distance from SEN for casting speed $1.4 \mathrm{~m} / \mathrm{min}$ and mold width (a) $1200 \mathrm{~mm}$ and (b) $1800 \mathrm{~mm}$. 


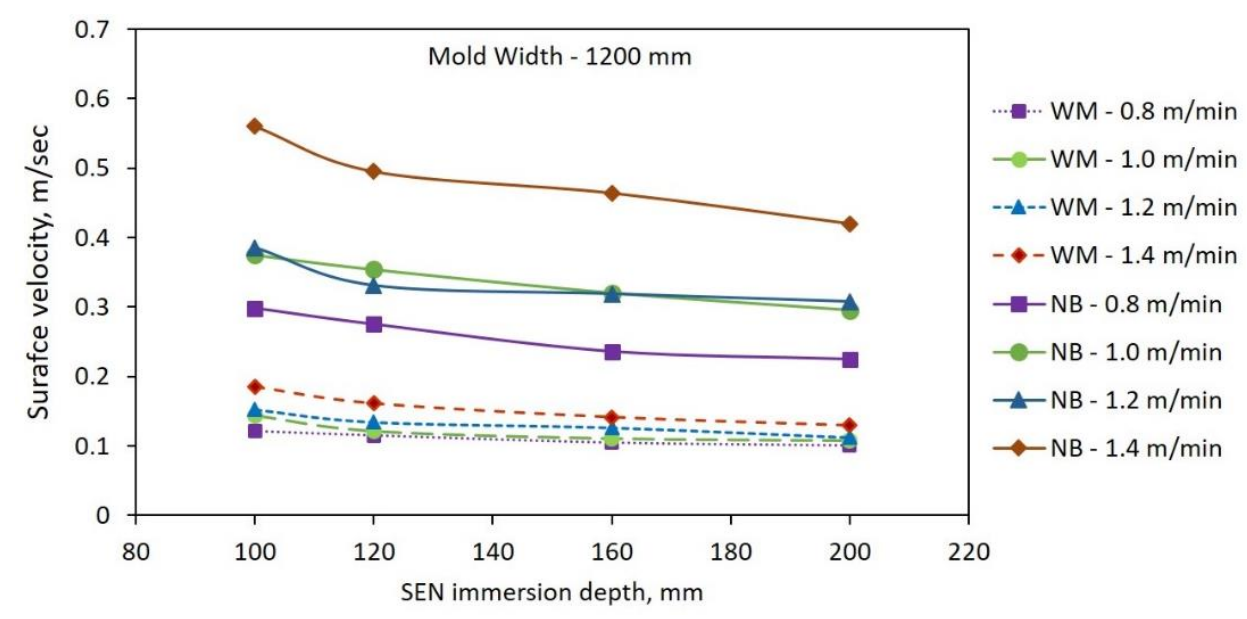

Fig. 14. Comparison of surface velocities obtained from water model (WM) and nail board (NB) experiments for mold width $1200 \mathrm{~mm}$.

A safe operating window to prevent mold slag entrapment during continuous casting was identified for mold widths 1200, 1400, 1600, and $1800 \mathrm{~mm}$ from the results of water modelling and nail board experiments and presented in Figs 15 \& 16. In the case of 1200 and $1400 \mathrm{~mm}$ mold widths, as shown in Fig. 15, higher casting speeds greater than or equal to $1.4 \mathrm{~m} / \mathrm{min}$ should be avoided to reduce too strong double roll flow and subsequent mold slag entrapment. Casting speeds lower than $1 \mathrm{~m} / \mathrm{min}$ should also be avoided to eliminate mixed flow and defects due to hook formation. Stable double roll flow with an optimized and medium surface flow velocity is superior to single roll and unstable flow to prevent mold slag entrapment. Therefore, for 1200 and $1400 \mathrm{~mm}$ mold widths, casting speed $1.0-1.3 \mathrm{~m} / \mathrm{min}$ is recommended to be maintained during continuous casting operation for SEN immersion depths $100-200 \mathrm{~mm}$ to avoid mold slag entrapment. 

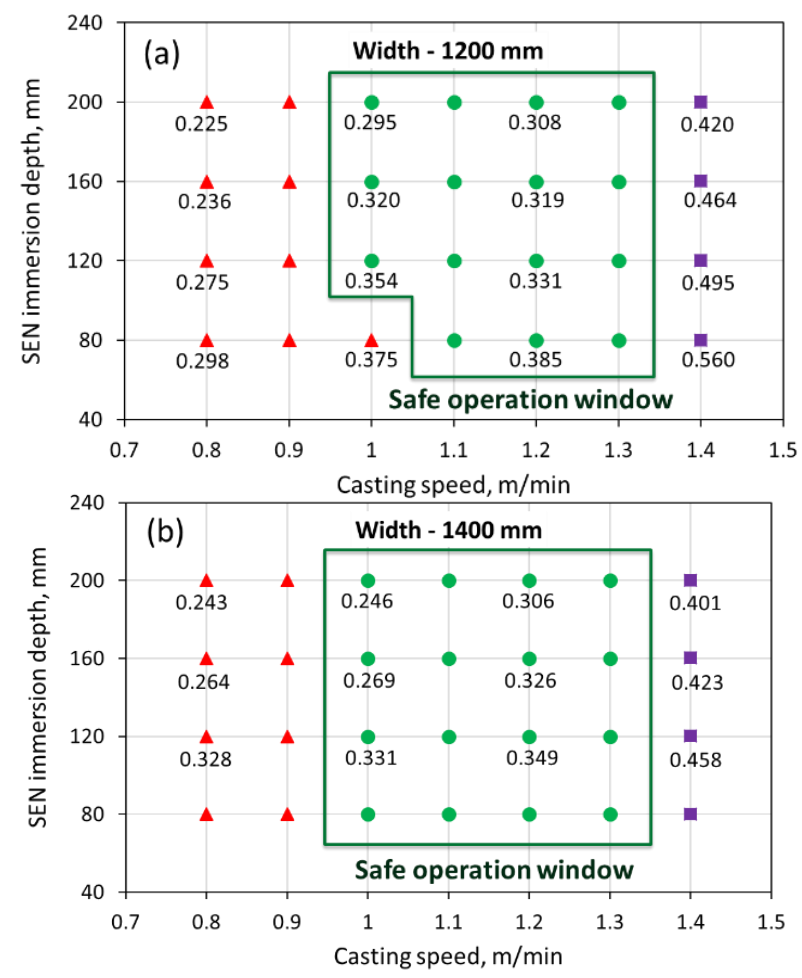

Fig. 15. Safe operation window to avoid mold slag entrapment for mold width (a) 1200 and (b) $1400 \mathrm{~mm}$ ( $\mathbf{\Delta}$ Unsteady flow, • Double roll flow-No slag entrapment, - Double roll flow-slag entrapment). Numerical values in the figure indicate surface (meniscus) velocities in $\mathrm{m} / \mathrm{sec}$.

For mold widths 1600 and $1800 \mathrm{~mm}$, optimum operating casting speed is 1.0-1.3 $\mathrm{m} / \mathrm{min}$ for SEN immersion depths $160 \& 200 \mathrm{~mm}$, and casting speed $1.0-1.1 \mathrm{~m} / \mathrm{min}$ is recommended for SEN depths $100 \& 120 \mathrm{~mm}$ as shown in Fig. 16. It can be seen from this figure that mold slag entrapment at lower casting speeds $(<1.0 \mathrm{~m} / \mathrm{min})$ is due to the unsteady flow of liquid steel in the mold. Mold slag entrapment was observed in this study at casting speeds 1.2-1.4 $\mathrm{m} / \mathrm{min}$ for shallow SEN immersion depths $100-120 \mathrm{~mm}$ and also at higher casting speed $1.4 \mathrm{~m} / \mathrm{min}$ for all SEN immersion depths as presented in Fig. 16 for mold widths 1600 and $1800 \mathrm{~mm}$. Higher casting speeds promote mold slag entrapment due to higher slag-metal interfacial drag force created by higher meniscus (surface) velocities. Surface velocity and mold level fluctuations increase with a decrease in SEN submergence depth that creates favorable conditions for mold slag entrapment. The critical surface velocity that promotes mold slag entrapment found from this study is $\sim 0.4$ $\mathrm{m} / \mathrm{sec}$ as indicated in Figs. 15 and 16 for mold widths 1200-1800 mm. Surface velocities greater than this critical value promote turbulent and unsymmetrical flow in the mold that lead to mold slag entrapment by interfacial shear mechanism. The critical amplitude of mold level fluctuations $(\delta)$ in the mold is related with this critical velocity as $\delta=35\left(\mathrm{~V}_{\mathrm{cr}}\right)$, where, $\mathrm{V}_{\mathrm{cr}}$ is critical surface velocity [2]. Therefore, it was calculated from this relation 
that the mold level fluctuations with amplitude greater than $14 \mathrm{~mm}$ promote mold slag entrapment and hence should be avoided. These results are in agreement with Hibbeler and Thomas [2]. Lower surface velocities resulted from lower casting speeds and too deep SEN immersion depths may cause meniscus freezing and promote the formation and growth of hooks $[2,4,11]$.
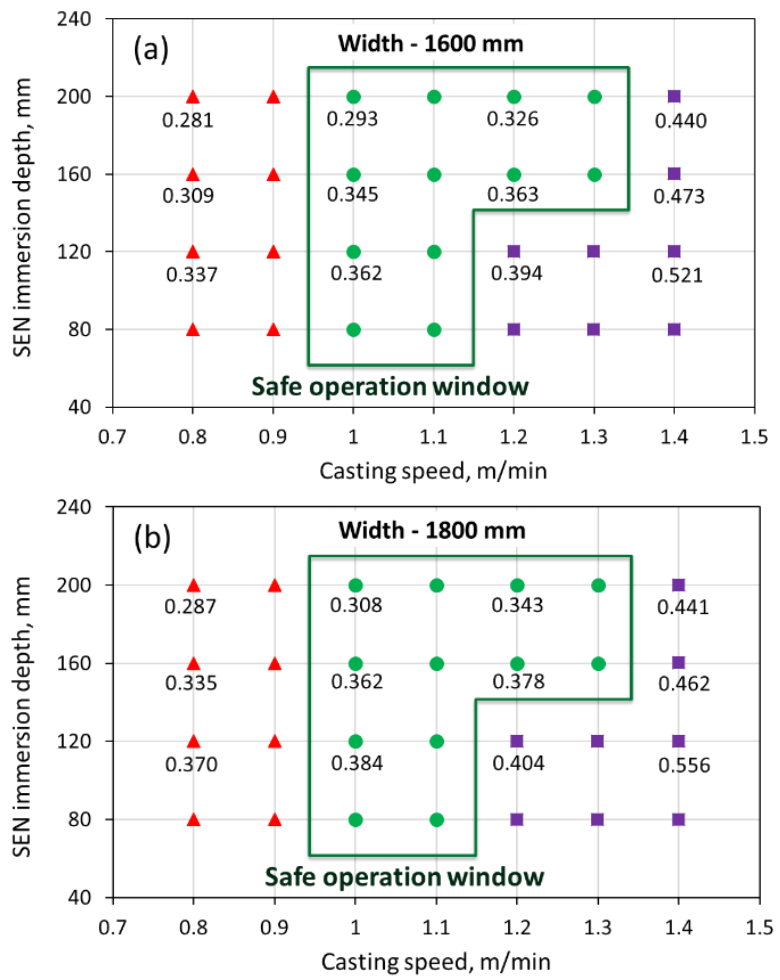

Fig. 16. Safe operation window to void mold slag entrapment for width (a) 1600 and (b) $1800 \mathrm{~mm}$ ( $\mathbf{\Delta}$ Unsteady flow, • Double roll flow-No slag entrapment, - Double roll flow-slag entrapment). Numerical values in the figure indicate surface (meniscus) velocities in $\mathrm{m} / \mathrm{sec}$.

\section{Conclusions}

The effect of SEN immersion depth and casting speed on mold slag entrapment in continuous caster mold was studied by water modeling and nail board experiments. SEN immersion depth and casting speed have a greater effect on mold slag entrapment. The following conclusions could be drawn from the study.

Optimum conditions of casting speed and SEN immersion depth for mold widths 1200-1800 mm were identified by water modeling and nail board experiments to avoid mold slag entrapment.

Surface (meniscus) velocity for lower mold widths is nearly unchanged up to casting speed $1 \mathrm{~m} / \mathrm{min}$ and then increases for all SEN immersion depths. However, for 
higher mold widths, surface velocity increases continuously with the increase in casting speed.

Liquid flow velocity at the mold surface greatly affects the tendency for mold slag entrapment. Higher surface velocities contribute to surface turbulence, vortex formation, and mold slag get trapped by interfacial shear mechanism, whereas low surface velocities promote mold slag entrapment by hook formation.

Critical surface velocity and critical amplitude of mold level fluctuations to avoid mold slag entrapment were identified in this study for the continuous casting of steel.

\section{References}

[1] F. Peng, Y. Min, C.J. Liu, M.F. Jiang: Int. J. Minerals, Metallurgy and Materials, 26 (2019) 186-193.

[2] C.L. Hibbeler, G.B. Thomas: AISTech Proceedings, (2013) 1215-1230.

[3] Dipak Mazumdar, I.L.G. Roderick: ISIJ Int., 39 (1999) 524-547.

[4] L. Zhang, B.G. Thomas: XXIV National Steelmaking Symposium, Morelia, Mexico, 26-28, Nov 2003, 138-183.

[5] X. Deng, C. Ji, Y. Cui, L. Li, X. Yin, Y. Yang, A. McLean: Iron Making and Steel Making, 44 (2017) 461-471.

[6] B. Rietow, B.G. Thomas: May 5-8, AISTech 2008, Pittsburgh, PA.

[7] R. Liu, B.G. Thomas, S. Joydeep, D.C. Stephen, M. Trinh: ISIJ Int., 54 (2014) 2314-2323.

[8] Z. Peng, Z. Lanhua: Iron Making and Steel Making, 46 (2019) 886-895.

[9] X. Li, B. Li, Z. Liu, R. Niu, Y. Liu, C. Zhao, C. Huang, H. Qiao, T. Yuan: Metals, MDPI, 9 (2019) 7-19.

[10] F. Wang, Y. Wang, S. Yang, W. Zhang, J. Li, X. Zhang: Contributed papers from Materials Science and Technology, MS\&T 2017, October, 2017, Pittsburgh, Pennsylvania USA.

[11] Q. Lu, R. Yang, X. Wang, J. Zhang, W. Wang: J. University of Science and Technology Beijing, 14 (2007) 399-404.

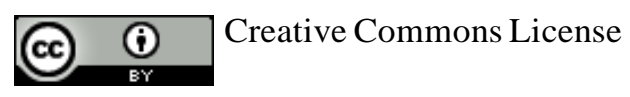

This work is licensed under a Creative Commons Attribution 4.0 International License. 